\title{
Counterpropagating frequency mixing with terahertz waves in diamond
}

\author{
Matteo Clerici,${ }^{1,2, *}$ Lucia Caspani, ${ }^{1}$ Eleonora Rubino, ${ }^{1,3}$ Marco Peccianti, ${ }^{4}$ Marco Cassataro,${ }^{1,5}$ \\ Alessandro Busacca, ${ }^{5}$ Tsuneyuki Ozaki, ${ }^{1}$ Daniele Faccio, ${ }^{2}$ and Roberto Morandotti ${ }^{1}$ \\ ${ }^{1}$ Institut National de la Recherche Scientifique - Énergie Matériaux Télécommunications (INRS-EMT), 1650 Blvd. Lionel-Boulet, \\ Varennes, Québec J3X 1S2, Canada \\ ${ }^{2}$ School of Engineering and Physical Sciences, Heriot-Watt University, Scottish Universities Physics Alliance (SUPA), Edinburgh EH14 4AS, UK \\ ${ }^{3}$ Dipartimento di Scienza e Alta Tecnologia, Università degli Studi dell'Insubria, via Valleggio 11, Como 22100, Italy \\ ${ }^{4}$ Institute for Complex Systems, Consiglio Nazionale delle Ricerche (CNR), via dei Taurini 19, Roma 00185, Italy \\ ${ }^{5}$ Dipartimento di Ingegneria Elettrica, Elettronica e delle Telecomunicazioni (DIEET), Università di Palermo, viale delle Scienze 9, \\ Palermo 61-90133, Italy \\ ${ }^{*}$ Corresponding author: clerici@emt.inrs.ca
}

\begin{abstract}
Frequency conversion by means of Kerr nonlinearity is one of the most common and exploited nonlinear optical processes in the UV, visible, IR, and mid-IR spectral regions. Here we show that wave mixing of an optical field and a terahertz wave can be achieved in diamond, resulting in the frequency conversion of the terahertz radiation either by sum- or difference-frequency generation. In the latter case, we show that this process is phase matched and most efficient in a counterpropagating geometry.
\end{abstract}

Terahertz radiation covers the spectral range between 0.1 and $10 \mathrm{THz}(3 \mathrm{~mm}$ to $30 \mu \mathrm{m})$ and is gathering an increasing interest both for spectroscopic applications and as a playground for fundamental studies, for instance on nonlinear and extreme-nonlinear optical effects [ $\underline{1}-\underline{5}]$. Furthermore, terahertz radiation is also attracting attention for its possible application, for example, as a control field for integrated nonlinear optics [6].

Although several studies have already investigated the wave mixing of terahertz and optical fields via the Kerr $-\chi^{(3)}$-nonlinearity, especially in gases (see, e.g., $[\underline{7}, \underline{8}]$ ), only a few have addressed the possibility of performing nonlinear wave mixing in bulk samples, typically exploiting electric-field-induced second harmonic generation [9-11] and, more recently, four-wave mixing for terahertz wave generation $[\underline{12}, \underline{13}]$.

In this Letter, we report on the wave mixing of terahertz and near-infrared radiation in a $\langle 100\rangle$-cut diamond bulk sample. We show that two processes, namely sumfrequency (SF) and difference-frequency (DF) generation, coexist, and that counterpropagating DF generation, taking place for an optical pulse interacting with a counterpropagating terahertz field, appears to be the most efficient process thanks to the longer coherence length.

We start by considering the SF and DF interactions:

$$
\mathrm{SF}: 2 \omega_{p}+\omega_{T}=\omega_{\mathrm{SF}} \mathrm{DF}: 2 \omega_{p}=\omega_{T}+\omega_{\mathrm{DF}},
$$

where $\omega_{p}$ is the optical pump frequency (in our case corresponding to a $792 \mathrm{~nm}$ wavelength), $\omega_{T}$ is the seed terahertz field carrier frequency, and $\omega_{\mathrm{SF}} / \omega_{\mathrm{DF}}$ is the frequency of the idler wave resulting from the SF/DF process (from here on we shall refer to SF/DF for both the effect and the generated field). Considering the case of a collinear interaction of plane, monochromatic waves, the phase-matching condition reduces to a scalar equation for the involved wavevectors. In this case, for both processes two different configurations are possible:
SF- $P: k_{\mathrm{SF}}=2 k_{p}+k_{T} \rightarrow \Delta k_{\mathrm{SF}-P}=k_{\mathrm{SF}}-2 k_{p}-k_{T}$

SF- $C: k_{\mathrm{SF}}=2 k_{p}-k_{T} \rightarrow \Delta k_{\mathrm{SF}-C}=k_{\mathrm{SF}}-2 k_{p}+k_{T}$

DF-P: $k_{\mathrm{DF}}+k_{T}=2 k_{p} \rightarrow \Delta k_{\mathrm{DF}-P}=k_{\mathrm{DF}}-2 k_{p}+k_{T}$

$\mathrm{DF}-C: k_{\mathrm{DF}}-k_{T}=2 k_{p} \rightarrow \Delta k_{\mathrm{DF}-C}=k_{\mathrm{DF}}-2 k_{p}-k_{T}$,

where $k_{i}$ denotes the $i$ th field wavevector ( $i$ being $p$ for the pump and $T$ for the terahertz field, with SF and DF as stated above) whereas $P$ and $C$ indicate the co- and counterpropagating configurations, respectively, and $\Delta k$ is the phase mismatch.

It is worth noting that in a dispersive medium the phase mismatch $\Delta k$ for the four interactions in Eq. (2) is different because $k_{\mathrm{SF}} \neq k_{\mathrm{DF}}$, as the two frequencies $\omega_{\mathrm{SF}}$ and $\omega_{\mathrm{DF}}$ are different.

In our experimental configuration, the terahertz pulse is generated by laser-induced plasma and shows peak electric fields in the order of few megavolts per centimeter with a duration of 90 fs (full width at halfmaximum). The details of the source are reported elsewhere [14]. The instantaneous electric field and bandwidth, measured by air-biased coherent detection [15] are shown in Fig. 1. The optical pump, delivered by a Ti:sapphire amplifier, has a duration of $60 \mathrm{fs}$ (full width at half-maximum) and carrier wavelength of $792 \mathrm{~nm}$. The frequencies of the SF and DF fields $\left[\omega_{\mathrm{SF}}\right.$ and $\omega_{\mathrm{DF}}$ in Eq. (1)] corresponding to the $0-25 \mathrm{THz}$ seed bandwidth are overlaid in Fig. 1(b), right scale.

In order to observe the nonlinear wave mixing between a terahertz and an optical pulse, a suitable material featuring low absorption at all the wavelengths involved in the process is essential. Diamond is the perfect candidate, showing negligible absorption $\left(<1 \mathrm{~cm}^{-1}\right)$ both in the terahertz and in the far-infrared bandwidth, as well as at $800 \mathrm{~nm}$ and at the $\lambda_{\mathrm{SF} / \mathrm{DF}}$ wavelength $(\sim 400 \mathrm{~nm})[\underline{16}]$. Furthermore, the high nonlinear coefficient guarantees reasonable frequency conversion efficiencies [ $\underline{17}-\underline{19}]$. 

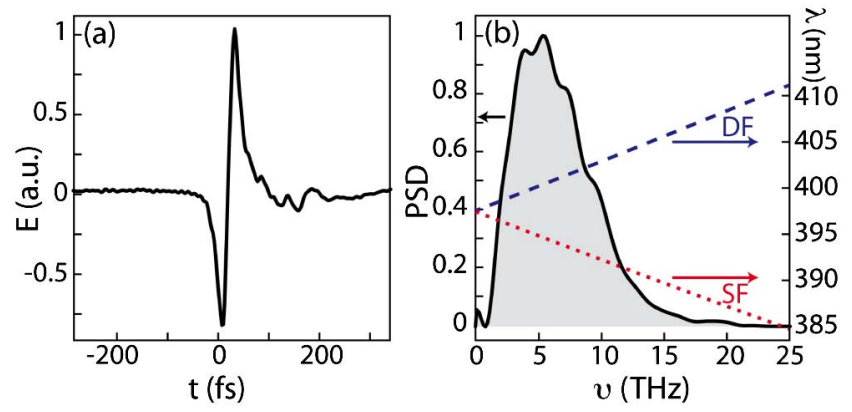

Fig. 1. (Color online) (a) Instantaneous electric field and (b) power spectral density for the terahertz pulse employed in our experiments. In (b), the dashed (blue) and dotted (red) lines show the DF and SF wavelengths corresponding to the terahertz seed frequency, respectively (right scale).

In our experiment, we have employed four different diamond samples. Two were single-crystal chemical vapor deposition slabs (Element Six TM) of 500 and $300 \mu \mathrm{m}$ thickness $(4.5 \mathrm{~mm} \times 4.5 \mathrm{~mm}$ and $3 \mathrm{~mm} \times 3 \mathrm{~mm}$ aperture, respectively). The others were two thinner polycrystalline films (Diamond Materials $\mathrm{GmbH}$ ) of 100 and $50 \mu \mathrm{m}$ thickness ( $5 \mathrm{~mm}$ clear aperture). In the first measurement, we investigated the copropagating geometry by overlapping, in the different diamond samples, the focused terahertz beam ( $\sim 90 \mu \mathrm{m}$ Gaussian beam waist) and a collimated $792 \mathrm{~nm}$ pump beam (1.5 mm beam waist). We hence recorded the SF/DF spectrum at different pump-to-terahertz time delays. A sketch of this configuration is shown in Fig. 2(a). Figure 2(b) shows the delay-resolved spectrum of the DF/SF for the $500 \mu \mathrm{m}$ thick sample. For the collinear, copropagating wave mixing between a $60 \mathrm{fs}$ (792 $\mathrm{nm}$ ) and a $90 \mathrm{fs}$ (terahertz) pulse, we would expect a delay-dependent signal lasting around 110 fs. On the contrary, the experimental results clearly show a trace far more extended, with a duration of nearly 8 ps [Fig. 2(b)], a value that is not consistent with the assumption of a purely copropagating geometry.
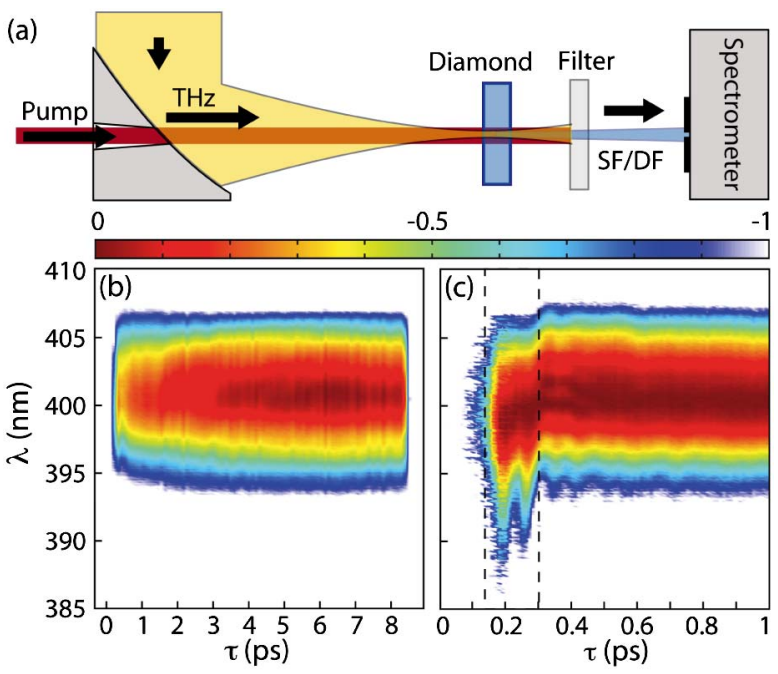

Fig. 2. (Color online) (a) Sketch of the experiment investigating the copropagating wave-mixing geometry. (b) Logarithmic representation of the wave-mixing spectrogram (normalized) in the violet spectral region. (c) Zoom of (b) on the delay region where copropagating wave mixing takes place. Note that (c) is normalized in a different way than (b); see text for details.
Considering only the initial region (close to the zerodelay), we clearly observe that the recorded signal is composed of two contributions. In Fig. 2(c) we highlight this by showing the zoomed spectrum up to a 1 ps delay, normalized to unit at each delay (for signals above 0.1 of the maximum recorded one, that is, where the signal-to-noise ratio is acceptable).

The first contribution, delimited by the vertical dashed lines in Fig. 2(c), originates from the copropagating process. For longer delays, a redshifted signal is observed lasting for much longer times. In order to understand the origin of this signal in this case, we show the coherence length $L_{c} \equiv \pi /|\Delta k|$ for the four different possible interaction geometries considered in Eq. (2) [Fig. 3(a)]. The solid blue and red curves are for the copropagating $\mathrm{DF}$ and SF, respectively. We note that the coherence lengths of these two processes are extremely small and comparable. The SF and DF frequencies can be extracted from the dotted and dashed lines in Fig. 1(b). Their temporal phases are determined by $2 \phi_{p} \overline{+\phi_{T}}$ and $2 \phi_{p}-\phi_{T}$, respectively, and the beating of these two signals has a component at twice the terahertz carrier frequency, which appears indeed at the shorter wavelengths in Fig. 2(c), as a function of the delay. The recorded SF component is, however, weaker with respect to the DF component (see also [20]).

The long-lasting, redshifted signal can thus be interpreted as the result of a more efficient backward phasematched interaction-DF-C, seeded by the terahertz signal (16.6\%) reflected from the output face of the diamond sample. From a simple analysis of the coherence lengths for the counterpropagating geometries, we note that the DF-C is perfectly phase-matched for a $10 \mathrm{THz}$ seed [dashed blue curve in Fig. 3(a)] while the SF-C is phase-mismatched (dashed red curve). The redshift is simply a consequence of the frequency matching shown by the blue dashed line in Fig. 1(b) for the phase-matched terahertz bandwidth (around $9.9 \mathrm{THz}$, which corresponds to $401.2 \mathrm{~nm}$ ). On the other hand, the asymmetry in the spectrum stems from the competing trends of the spectral power density peaked at $\simeq 5 \mathrm{THz}$ and of the phase matching fulfilled at $\simeq 10 \mathrm{THz}$.

The counterpropagating phase-matching hypothesis is confirmed by the analysis of the delay-dependent DF
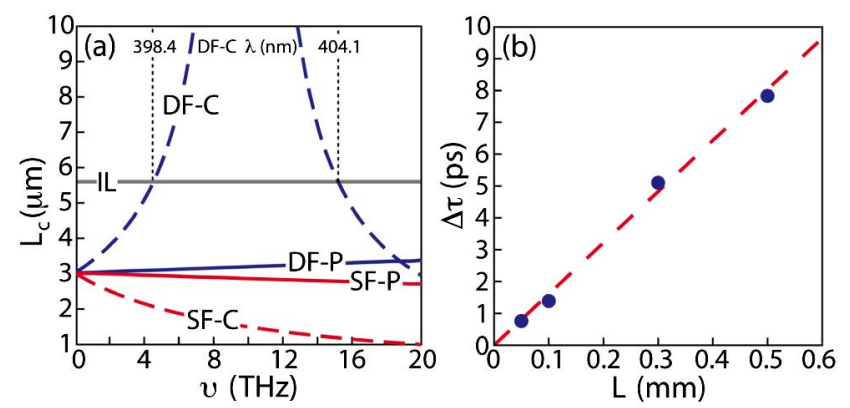

Fig. 3. (Color online) (a) Coherence lengths as a function of the frequency for the co- (solid) and counter- (dashed) propagating $\mathrm{SF}$ (red) and DF (blue) processes. The gray horizontal line is the estimated DF-C interaction length (IL). The wavelength on top shows the DF-C bandwidth. (b) The dots show the experimentally recorded duration $(\Delta \tau)$ of the DF signal for different crystal thicknesses $L$. The dashed line is the duration calculated from the pulses' group velocities (see text for details). 

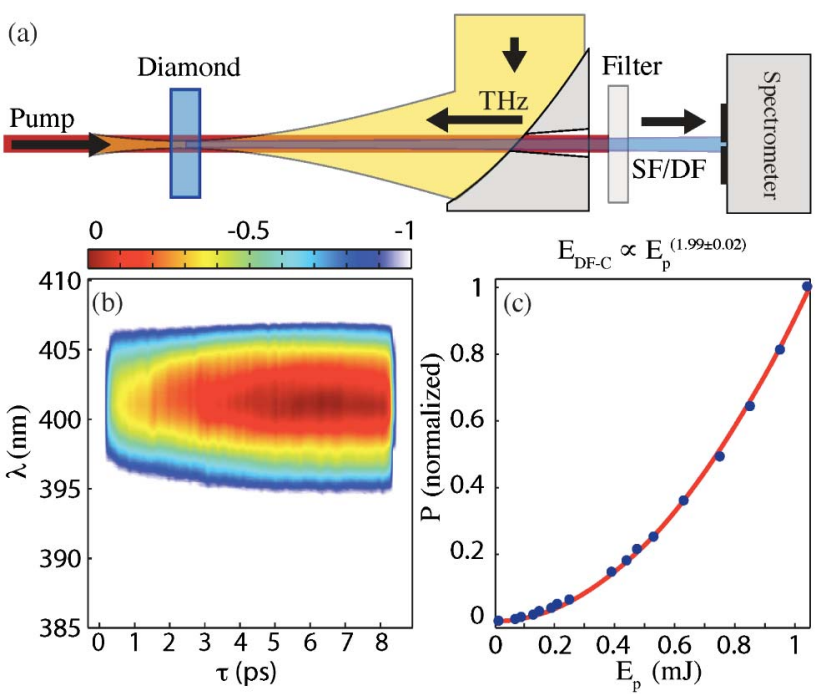

Fig. 4. (Color online) (a) Sketch of the experiment investigating the counterpropagating geometry. (b) Spectrogram of the wave mixing in the violet spectral region (log scale, normalized). (c) Normalized DF-C power as a function of the pump energy (blue dots). The red curve is a power fit with exponent $1.99 \pm 0.02$.

signals recorded for the four different sample thicknesses. In a counterpropagating geometry the delaydependent signal is expected to extend along the delay coordinate $\tau$, for $\Delta \tau \approx L\left(1 / v_{g, T}+1 / v_{g, p}\right)$, where $v_{g, T / p}$ are the terahertz and optical pulse group velocities, respectively. In our experiments, the recorded $\Delta \tau$ values for the four samples [blue dots in Fig. 3(b)] match what is predicted analytically (red dashed line). Noteworthy, no difference in the signal duration is expected between single-crystal and polycrystalline samples of equal thicknesses. This was verified by comparing measurements performed on either polycrystalline or single-crystal $500 \mu \mathrm{m}$ thick diamond samples.

In order to further confirm our conclusions and to characterize the DF-C signal in the phase-matched geometry interacting with the whole input seed rather than just a reflection, we performed a second measurement, directly injecting the terahertz pulse counterpropagating with respect to the $792 \mathrm{~nm}$ pump pulse in the $500 \mu \mathrm{m}$ diamond sample [see sketch in Fig. 4(a)]. The DF-C signal is spectrally resolved for different pump-terahertz delays, resulting in a spectrogram [Fig. 4(b)] similar to the one measured in the previous configuration [Fig. 3(b)], except for the absence of the initial blueshifted part, further confirming the counterpropagating phase-matching hypothesis.

Finally, we recorded the generated DF-signal power in the counterpropagating geometry for different pump pulse energies, reported in Fig. 4(c). A power fit confirms the expected quadratic dependence. The recorded low values for the DF-signal power are mainly a consequence of the short interaction length (IL), limited by the reduced pulse overlap $(\sim 5.5 \mu \mathrm{m})$ due to the counterpropagating geometry: IL $=\tau_{T}\left(1 / v_{g, p}-1 / v_{g, T}\right)$ (solid gray line in Fig. 3).

In conclusion, we have shown, for the first time to the best of our knowledge, a wavelength-shifting mechanism (see, e.g., [21,22]) relying on a naturally phase-matched
DF generation process occurring in a Kerr medium (diamond) between counterpropagating waves.

Several intriguing applications can be envisaged, such as the detection and imaging of terahertz fields in a counterpropagating geometry. Furthermore, our results hint toward further investigations of counterpropagating wave-mixing [23].

MC acknowledges the support of the IOF People Programme (Marie Curie Actions) of the European Union's FP7-2012, KOHERENT, GA 299522. LC and ER acknowledge the support from "Le Fonds québécois de la recherche sur la nature et les technologies." The authors acknowledge AXIS Photonique Inc., M. Bouvier, and the ALLS staff for technical support; P. Di Trapani, G. Assanto and F. Légaré, for enlightening discussions; and B. E. Schmidt and N. Thiré for experimental support.

\section{References}

1. G. P. Williams, Rep. Prog. Phys. 69, 301 (2006).

2. K. Reimann, Rep. Prog. Phys. 70, 1597 (2007).

3. W. L. Chan, J. Deibel, and D. M. Mittleman, Rep. Prog. Phys. 70, 1325 (2007).

4. M. Tonouchi, Nat. Photonics 1, 97 (2007).

5. M. Liu, H. Y. Hwang, H. Tao, A. C. Strikwerda, K. Fan, G. R. Keiser, A. J. Sternbach, K. G. West, S. Kittiwatanakul, J. Lu, S. A. Wolf, F. G. Omenetto, X. Zhang, K. A. Nelson, and R. D. Averitt, Nature 487, 345 (2012).

6. A. Novitsky, M. Zalkovskij, R. Malureanu, P. U. Jepsen, and A. V. Lavrinenko, Opt. Lett. 37, 3903 (2012).

7. J. Dai, X. Xie, and X. C. Zhang, Phys. Rev. Lett. 97, 103903 (2006).

8. J. Dai, X. Xie, and X. C. Zhang, Appl. Phys. Lett. 91, 211102 (2007).

9. C. Ohlhoff, C. Meyer, G. Lüpke, T. Löffler, T. Pfeifer, H. G. Roskos, and H. Kurz, Appl. Phys. Lett. 68, 1699 (1996).

10. A. Nahata and T. F. Heinz, Opt. Lett. 23, 67 (1998).

11. D. Cook, J. Chen, E. A. Morlino, and R. M. Hochstrasser, Chem. Phys. Lett. 309, 221 (1999).

12. K. Suizu and K. Kawase, Opt. Lett. 32, 2990 (2007).

13. Z. Wang, H. Liu, N. Huang, Q. Sun, and J. Wen, Opt. Express 20, 8920 (2012).

14. M. Clerici, M. Peccianti, B. E. Schmidt, L. Caspani, M. Shalaby, M. Giguère, A. Lotti, A. Couairon, F. Légaré, T. Ozaki, D. Faccio, and R. Morandotti, "Scaling mechanism for efficient wavelength conversion in laser plasmas," ArXiv 1207.4754 (2012).

15. N. Karpowicz, J. Dai, X. Lu, Y. Chen, M. Yamaguchi, H. Zhao, X.-C. Zhang, L. Zhang, C. Zhang, M. Price-Gallagher, C. Fletcher, O. Mamer, A. Lesimple, and K. Johnson, Appl. Phys. Lett. 92, 011131 (2008).

16. A. M. Zaitsev, Optical Properties of Diamond (Springer, 2001).

17. R. Boyd, Nonlinear Optics (Academic, Elsevier, 2008).

18. M. Levenson and N. Bloembergen, Phys. Rev. B 10, 4447 (1974).

19. J. Zhao, G. Jia, X. Liu, Z. Chen, J. Tang, and S. Wang, Chin. Opt. Lett. 8, 685 (2010).

20. X. Lu, N. Karpowicz, and X. C. Zhang, J. Opt. Soc. Am. B 26, A66 (2009).

21. K. Gallo, G. Assanto, and G. I. Stegeman, Appl. Phys. Lett. 71, 1020 (1997)

22. C. Conti, A. Di Falco, and G. Assanto, Opt. Express 12, 823 (2004).

23. Y. Ding, S. Lee, and J. Khurgin, Phys. Rev. Lett. 75, 429 (1995). 